\title{
LA ÉTICA DEL CUIDADO EN EL CONTEXTO DE LA SALUD SEXUAL Y REPRODUCTIVA
}

\author{
Gabriel Jaime Montoya Montoya*
}

\begin{abstract}
Resumen: La ética del cuidado es un constructo teórico y práctico que busca resaltar la vinculación esencialmente humana y emocional entre el profesional de la salud y el paciente. En este artículo se propone su instrumentación en el campo de la salud sexual y, principalmente, en la difusión y establecimiento de los derechos sexuales. Dicha estrategia está orientada a propiciar el diálogo bioético entre los profesionales de la salud y las personas que reciben su cuidado, puesto que la reciprocidad de conocimientos e información genera empatía y trato humanizado. Se consideran la definición de salud sexual, la declaración de los Derechos Sexuales y la ética de la sexualidad como elementos que se integran finalmente en la ética del cuidado de las personas que acuden a los servicios de salud en el contexto latinoamericano.
\end{abstract}

Palabras clave: ética del cuidado, ética de la sexualidad, salud sexual, derechos sexuales

\section{ETHICS OF CARE IN SEXUAL AND REPRODUCTIVE HEALTH CONTEXT}

\begin{abstract}
Ethics of care is a theoretical and practical construct which highlights the essentially human and emotional bond between the health care professional and the patient. This essay emphasizes its application to the field of reproductive health and, mainly, to the dissemination and setting of the reproductive rights. Such strategy is oriented towards allowing a bioethics dialogue among health care professionals and whoever receives their care, since knowledge and information reciprocity generates empathy and humane treatment. The definition of sexual health, the Declaration of Sexual Rights and sexual ethics are considered integrated elements in the ethics of care for those looking for health care services in the Latin American context.
\end{abstract}

Key words: ethics of care, ethics of sexuality, reproductive health, reproductive rights

\section{A ÉTICA DO CUIDADO NO CONTEXTO DA SAÚDE SEXUAL E REPRODUTIVA}

Resumo: A ética do cuidado é um construto teórico e prático que busca ressaltar a vinculação essencialmente humana e emocional entre o profissional da saúde e o doente. Neste artigo se propóe sua instrumentalização no campo da saúde sexual e, principalmente, na difusão e estabelecimento dos direito sexuais. Tal estratégia orienta-se na perspectiva de propiciar o diálogo bioético entre os profissionais de saúde e as pessoas que recebem seu cuidado, posto que a reciprocidade de conhecimentos e informação gera empatia e trato humanizado no cuidado em saúde. São consideradas a definiçẫo de saúde sexual, a declaração dos Direitos Sexuais e a ética da sexualidade, como elementos que se integram finalmente na ética do cuidado, das pessoas que procuram os serviços de saúde no contexto latinoamericano.

Palavras-chave: ética do cuidado, ética da sexualidade, saúde sexual, direitos sexuais

* Médico Psiquiatra. Grupo de Investigación en Psiquiatría, Facultad de Medicina, Universidad de Antioquia, Colombia Correspondencia: gajamon2004@yahoo.com 


\section{Introducción}

Desde sus orígenes, la bioética ha indagado por las claves que definen la vinculación entre médico y paciente. A esta díada clásica se han añadido progresivamente varios agentes que triangulan las relaciones: los avances tecnológicos, el reconocimiento legal e institucional de los derechos de los pacientes, la implementación de las políticas de calidad y, en general, la transformación de los sistemas generales de seguridad social.

Una de las quejas más repetidas entre las comunidades de usuarios radica en la pérdida de cercanía con el terapeuta. La posibilidad de un trato humanizado se desdibuja en medio de las presiones por la optimización de recursos que, paradójicamente, buscan garantizar la universalización de la atención en el contexto de la justicia social. Las reformas a los sistemas de salud y de seguridad social, en general, han motivado frecuentes discusiones en los países de América Latina y el Caribe. Si bien se buscan estrategias para la atención oportuna, se producen en forma colateral reflexiones sobre la calidad de la misma.

El cuerpo de conocimientos sobre lo sexual ha crecido en forma asimétrica y en una menor proporción que otros frentes de las ciencias de la salud. Reconocer que es necesario, conveniente y justo indagar por la sexualidad de las personas y las poblaciones ha significado una ruptura paradigmática que, se espera, lleve a la reflexión en el personal de salud sobre sus carencias de conocimiento y capacitación. No obstante, también alude a la profunda necesidad de dar a conocer a la población el derecho a la salud sexual.

La atención sanitaria recibe personas con necesidades latentes o manifiestas en su sexualidad. Los ámbitos clásicos y vigentes de la prevención de enfermedades de transmisión sexual y de embarazos no deseados han venido albergando, cada vez más, a personas que vienen a consultar por disfunción eréctil o bajo deseo sexual, entre otras disfunciones. En los países latinoamericanos esta realidad se ha mezclado con los devastadores efectos de la violencia sexual y el maltrato infantil. En consecuencia, cuando se propone hablar de sexualidad y de salud sexual se alude a una realidad sustancial y visceral de nuestras sociedades.

En tales circunstancias, la ética del cuidado puede convertirse en una estrategia que permita sensibilizar al personal de salud acerca de la realidad de las personas que acuden a solicitar sus servicios. Igualmente, ofrece la opción de enfrentamientos asertivos y "empoderados" en las personas que experimentan amenazas o daños en su salud sexual. Dicho acercamiento no está circunscrito necesariamente a una visión particular de género o a la instalación de una dicotomía entre una atención en salud basada en el paradigma de lo femenino o lo masculino.

Este artículo se inicia con un recorrido por la ética de la sexualidad; luego, introduce los elementos de la ética del cuidado y su evolución conceptual, y, finalmente, presenta su vinculación con los derechos sexuales. Busca demostrar que la aproximación a la salud sexual y reproductiva desde la ética del cuidado puede ser una herramienta efectiva para garantizar el trato humanizado en la atención en salud.

\section{Ética de la sexualidad}

En la ética naturalista de la sexualidad, los actos son intrínsecamente buenos cuando respetan el orden interno de la naturaleza e intrínsecamente malos cuando no lo respetan. Además, la sexualidad se hace moral cuando se ejercita con moderación. Para esta ética, la naturaleza tiende siempre a un fin (telos) y todo órgano tiene también uno. Para el caso de los órganos de la generación, sus fines son la reproducción y perpetuación de la especie, de tal manera que los usos no dirigidos a ellos serían inmorales. La virtud que acompaña el ejercicio de la vida sexual es la templanza, el justo medio aristotélico; por consiguiente, el exceso o abstinencia serían inmorales. La templanza tiene por objeto regular el uso de los placeres corporales, principalmente el tacto y el gusto(1).

La visión cristiana de la sexualidad adoptó estos elementos naturalistas de la sexualidad; no obstante, debió resolver el asunto de la abstinencia sexual como un acto inmoral. Sustituyó entonces la ética por una ascética de la sexualidad: el hombre es un sujeto pasivo de sus pasiones, a diferencia de Dios que no tiene pasiones y es agente. El hombre sabio debe ser imitador de Dios $y$, en consecuencia, debe dedicarse a anular completamente los afectos (pathos) y las pasiones por la vía de la abstinencia. Quedaba así resuelto el asunto de la inmoralidad de ésta.

Esta elaboración concluía que son deshonestas, es decir no se hayan bajo el control de la razón de un hombre sabio, todas las prácticas sexuales que alteran el orden de la naturaleza. Para Tomás de Aquino éstas serán más 
graves cuanto más contravengan el orden determinado por la naturaleza. Para demostrarlo construye una escala de vicios contra naturaleza donde los menos graves son el incesto, la fornicación, el estupro, el adulterio y el rapto de una virgen, mientras que los más graves son la masturbación, la bestialidad, la homosexualidad y las relaciones heterosexuales por vías distintas de las usuales, con lo que, según el razonar tomista, el sexo oral termina siendo más grave que el incesto.

Más adelante, la modernidad planteó una oposición entre lo natural y lo moral. El hombre pasó a ser por sí mismo la fuente de lo moral: el hombre y no la naturaleza determina lo bueno y lo malo. El ser humano se convierte en fuente de su propia moralidad, con la racionalidad como instrumento. La autonomía empieza a ser protagonista en el ámbito ético, con el respeto a la dignidad del ser humano como valor fundamental. Para la ética sexual de la modernidad, se obra adecuadamente cuando se actúa por deber, considerando a la otra persona como un fin y no solamente como un medio para el placer. La manera como esto se produce es por medio del vínculo, no sólo con el cuerpo o la sexualidad de la otra persona, sino con todo su ser íntegro.

En la modernidad, "la moralidad o inmoralidad de los actos dependerá del grado de respeto o agresión que supongan a la dignidad de los seres humanos, y no de su carácter natural o antinatural" (1). En consecuencia, los actos sexuales respetan la dignidad del ser humano cuando se realizan entre personas autónomas que actúan con conocimiento, voluntad y libertad.

Posteriormente, la llamada "revolución sexual" del siglo XX disolvió las conexiones entre sexualidad y reproducción, y consolidó el respeto humano como principio moral para los actos sexuales. De esta manera, se crea un nicho para el ingreso de la ética de la responsabilidad. Su propuesta es trascender la dicotomía entre naturaleza y razón porque ninguna puede dar cuenta completa de lo moral en sexualidad. La ética de la responsabilidad se ubica en un punto intermedio, en un proceso que Diego Gracia denomina de "ponderación ambivalente".

En el ámbito privado, el respeto a los seres humanos se concreta en los principios éticos de autonomía y beneficencia. Como la sexualidad es, en esencia, una actividad privada, "un acto será moralmente reprobable cuando viole la autonomía de las personas o éstas no lo consideren beneficioso para ellas". La no maleficencia y la justicia establecen los límites de la autonomía: "por maleficentes o por injustas, consideramos inmorales todas aquellas conductas que agreden, escandalizan, etc. a personas determinadas, sobre todo si son menores de edad o incapaces. La violación, la agresión sexual, el acoso sexual, el exhibicionismo y la provocación sexual, etc., son conductas moralmente negativas precisamente por eso".

Las conductas sexuales que atentan directamente contra el respeto a la dignidad humana están reguladas, en consecuencia, por los llamados deberes perfectos y generan un derecho correlativo para ser respetado en los demás seres humanos. Estos deberes se deben exigir a todos, incluso con el auxilio de la fuerza. Este es precisamente el ámbito del derecho en la sexualidad: identificar los deberes perfectos y hacerlos cumplir con la ley.

En el campo de la sexualidad, los deberes imperfectos pertenecen a la esfera de lo privado y se cumplen según el sistema de valores y el proyecto de vida particulares. No generan un derecho correlativo ni puede exigirse su cumplimiento por la fuerza. "El derecho no puede ni debe inmiscuirse en el ámbito de los deberes imperfectos, sino que su función es asegurar ese marco mínimo y universal que permita que los seres humanos lleven a cabo, diferencialmente, la gestión de sus propios deberes imperfectos, es decir, sus proyectos de vida, de acuerdo con sus sistemas de valores y con sus ideas de perfección y felicidad".

El asunto es que existe diversidad de proyectos vitales: múltiples individuos, con diversos proyectos; los "extraños morales" conviven en un mismo sitio y en un mismo momento histórico. Cada uno se rige por sus deberes imperfectos engendrados en la privacidad e individualidad de su sexualidad. La propuesta consiste, entonces, en elevar al rango de deber perfecto el respeto al pluralismo y a la libertad de conciencia. Todo individuo tiene derecho a disfrutar de su sexualidad siempre y cuando no atente contra el imperativo de respeto a la dignidad de los seres humanos. Las leyes han de promover entonces el respeto por la diversidad sexual, como expresión del pluralismo, y procurar los medios para el respeto de la opción sexual de todos los miembros de una sociedad.

Respecto del derecho y la ética de la responsabilidad en sexualidad, Diego Gracia concluye: "La sexualidad es, básicamente, un asunto privado, un deber imperfecto, que no puede ni tiene que estar rígidamente reglamen- 
tado por el Derecho. Éste lo único que puede hacer es prohibir aquellas conductas que atenten contra los derechos y libertades de los demás, es decir, los deberes que sí son perfectos y que, por tanto, generan derechos en los demás (...) el ejercicio de la sexualidad pertenece a la esfera de lo privado, más aún, de lo íntimo, y por tanto está protegido por el derecho a la privacidad y a la intimidad (...) el respeto a la diversidad sexual y al ejercicio libre de la sexualidad, por supuesto dentro de ciertos límites que las sociedades tienen que establecer por consenso, es un deber perfecto, generalmente conocido con los nombres de derecho a la intimidad y a la privacidad"(1).

La sexualidad y los derechos sexuales están supeditados a la ética de la responsabilidad. Ellos son instrumentos valiosos para la evaluación ética de las conductas sexuales dentro de la sociedad y el Derecho. Sin embargo, en el contexto particular de la atención en salud y en la promoción de los derechos sexuales, es pertinente introducir los elementos de la ética del cuidado, por las condiciones especiales de trato humano y cercanía personal que favorecen las acciones de promoción y prevención de la salud sexual.

\section{Ética del cuidado}

El término "cuidado" tiene dos vertientes de significado: por un lado, la percepción de amenaza o peligro frente a algún elemento del entorno; en este caso, "tener cuidado" se refiere a las precauciones para el mantenimiento de la salud y la disminución de un daño potencial. Por otro, la palabra se vincula con la vigilancia de alguien o algo que se encuentra en situación de vulnerabilidad, es decir, "cuidar de". Este último significado se asocia a la función médica y, tradicionalmente, al rol de la enfermería en la atención clínica.

La evolución de la salud pública, la epidemiología y la medicina preventiva ha introducido conceptos como "factor de riesgo" y "condiciones de vulnerabilidad". Su aplicación práctica supone el diseño de estrategias en las cuales las personas y los grupos humanos deben adoptar estilos de vida que resulten saludables y disminuyan el impacto de la enfermedad. Esta lógica refleja otra arista de significación para la palabra "cuidado", en tanto cada persona debe hacerse cargo de su propio bienestar y corregir los hábitos que aumentan su riesgo de enfermarse. Ya no sólo se trata de esperar qué hace el médico para cuidar el bienestar del paciente sino, también, cómo moviliza este último sus propios recursos para conservar y recuperar el estado de salud. Así, cada persona debe tener introyectada una noción de autocuidado que supera la visión tradicional del heterocuidado.

Rollo May introduce elementos de la filosofía existencialista en el contexto de la psicología humanista y la ética del cuidado(2). Frente a la adversidad e insensibilidad del mundo, surge el cuidado como un antídoto a esta apatía. El cuidado es el fenómeno constitutivo básico de la existencia humana, su asimilación requiere el reconocimiento del otro como un ser humano y la identificación con su dolor o su disfrute. May señala, además, que el cuidado de sí mismo precede al cuidado del otro: nadie puede hacerse cargo de la asistencia de otra persona si no tiene unas condiciones básicas que den cuenta de su propio bienestar.

Erik Ericsson, por su parte -en su teoría del desarrollo psicosocial que contempla ocho estadios del ciclo vital-, describe en el séptimo estadio, correspondiente a la adultez, la crisis generabilidad versus autoabsorción o estancamiento. Se entiende por generabilidad la capacidad y el interés por establecer, proteger y orientar la próxima generación. La virtud que emerge de esta crisis es el cuidado(3). En este autor se da una convergencia ética entre el cuidado y la responsabilidad, en tanto ambos conceptos responden al interés por el bienestar de las futuras generaciones.

Para Erikson, tal capacidad de cuidar del otro se refleja en la labor paterna pero, también, en la docencia, la producción y la asistencia. Esta ética del cuidado surge por la tensión de dos fuerzas en el curso del ciclo vital, de tal manera que su consolidación sería el resultado de un proceso esperado de desarrollo. El registro eriksoniano del cuidado contempla la característica de universalidad para el género humano y su ausencia es vista como un estancamiento o detención en la evolución moral de los individuos.

$\mathrm{Al}$ introducirse en el ámbito de la atención médica, la evolución conceptual de la ética del cuidado ha enfatizado en la competencia técnica o excelencia en el oficio: quien decide cuidar a otro debe saber, como insumo mínimo, las condiciones básicas de lo que hace. Este conocimiento del arte requiere una vinculación profunda con su desarrollo teórico y práctico. Sin embargo, en el siglo XX surgieron críticas al personal de salud por haberse concentrado exclusivamente en el esclarecimiento de las causas patológicas y las estrategias 
terapéuticas, abandonando el arte médico que atendía íntegramente al paciente y no solamente su enfermedad. Esto ha resultado en una visión despersonalizada de la medicina.

Uno de los abanderados de la necesidad del cuidado en la atención médica fue Francis Peabody, profesor de la Universidad de Harvard. En 1927 señaló la necesidad de estar atento a las condiciones particulares de cada paciente, mediante la revisión del tipo de relación que ambos sostienen. Este toque "personal", por así llamarlo, supera la perspectiva organicista y patologicista de la medicina moderna, y se convierte, además, en una herramienta útil y necesaria en todo proceso de curación. La relación debe tener elementos de cortesía, simpatía, afabilidad y cercanía personal(4).

Dicha perspectiva de la atención, que vislumbra una significativa vinculación emocional entre cuidador y paciente, requirió, a su vez, la transformación de la "simpatía" en "empatía", con el fin de evitar la excesiva vinculación, la generación de dependencia y la pérdida de los límites terapéuticos. La empatía ha sido definida como la capacidad cognitiva del médico - o cuidadorpara entender las necesidades del paciente, la sensibilidad afectiva para identificar los sentimientos de éste y la habilidad comportamental para demostrar que se han comprendido tales necesidades y sentimientos $(5,6)$. La simpatía hace alusión al estado en el cual una persona es afectada por la condición de otra, produciéndose un sentimiento similar o correspondiente. En su nivel más básico, la simpatía recurre al sentimiento primario que surge ante una situación: de ahí la carga emocional que lleva implícita. En estos casos, la evaluación ética está contaminada por marcados elementos subjetivos que alteran la conformación del marco básico para el diálogo.

Cuando la ética del cuidado recurre a la empatía lo hace también amparada en su estructura tripartita (cognitiva, emocional y comportamental). De tal manera que es posible aprender a ser empático y evaluar las conductas empáticas frente a una situación determinada.

Con Carol Gilligan, en la década de los 80 , se estructura el discurso que actualmente conocemos como "ética del cuidado" (ethics of care). Uno de sus esfuerzos ha sido desarrollar una ética filosófica y sistemática más allá del entorno de la práctica sanitaria(7). Gilligan sostiene que hombres y mujeres tienden a emplear estrategias diferentes de razonamiento y aplican distintas ideas y conceptos cuando formulan y resuelven problemas morales. Estas diferencias harían que las mujeres perciban los conflictos éticos en términos de apego o desapego personal; de este modo, la tendencia femenina sería la evitación del aislamiento y el daño, la generación de fuertes vínculos interpersonales y la tendencia a la acción a partir del reconocimiento individual de una responsabilidad con el otro. En el discernimiento moral femenino importan elementos como la confianza, la calidad de los cuidados y la sensibilidad ante la adversidad y el dolor de las personas.

En el terreno masculino, la tensión se basa en los principios de equidad e inequidad. En su pensamiento ético, los hombres recurren más al uso de ideales abstractos, como justicia y derecho, la primacía de los derechos individuales y las normas universales. Los dilemas morales son resueltos, entonces, a partir de jerarquías de valores y los elementos de justicia darían el soporte para decidir cuál es la mejor decisión en un conflicto. Gilligan llama a la orientación moral más prevalente en las mujeres "ética del cuidado" y a la de los hombres "ética de la justicia".

¿Cuál es la validez de esta distinción en el razonamiento ético? Tal vez el mismo proceso de identidad de género y la asimilación social del rol de género llevan a hombres y mujeres a razonar de una peculiar manera (la descrita por Gilligan, por ejemplo). Las teorías del aprendizaje también muestran cómo la capacidad cerebral y mental de aprender y desaprender varía según las condiciones del entorno.

En todo caso, resulta interesante reflexionar acerca del agente que participa en el juicio ético en cuanto variable significativa en el resultado final. Esto implica que la discusión trasciende la dicotomía "feminista versus machista" -con la que ha sido criticada la ética del cuidado- para hacer acopio de los datos disponibles y entender lo valioso de la diversidad del pensamiento cuando se considera el registro de género.

En la ética del cuidado se otorga un papel moral a las emociones; igualmente, se resalta que las decisiones éticas comprometen en forma significativa la afectividad y todas las esferas de la personalidad. En la acción de cuidar se parte de un hecho individual, como es el autocuidado, y se trasciende para atender a los que requieren ayuda para sus necesidades vitales. En consecuencia, se genera una reciprocidad que fortalece los vínculos sociales y la calidad de la atención. 
Complementando esta perspectiva, el modelo de relación de ayuda de Carol Taylor propone que el profesional (cuidador) debe reunir seis núcleos básicos:

- Afecto: experimentar un sentimiento positivo hacia el paciente. Desde la perspectiva psicoanalítica, el abordaje y análisis de la transferencia y contratransferencia.

- Cognición: tener la capacidad intelectual y técnica de identificar lo esencial para el bienestar del paciente.

- Volición: se refleja en el compromiso interno para movilizar los recursos personales y técnicos que aseguren el bienestar del paciente.

- Imaginación: emplear creativamente los recursos personales para relacionarse empáticamente con el paciente y entender su situación existencial particular, así como sus necesidades vitales.

- Motivación: corresponde al flujo de energía aplicado al acto de cuidar, refleja el altruismo en el ejercicio de la profesión y el deseo de actuar en consecuencia.

- Expresión: consiste en la habilidad para demostrar genuinamente la intención de ayuda mediante el uso de la estrategia de comunicación verbal y no verbal.

La ética del cuidado reflexiona sobre los conflictos entre responsabilidades. Su ámbito de acción es predominantemente el área de la salud, aunque no excluye su aplicación en todas las áreas que trabajan por el bienestar de las personas. Si bien surge a partir de observaciones respecto de los estilos femenino y masculino de abordaje de los conflictos éticos, logra superar la condición de género. La creación de confianza, la sensibilidad respecto del dolor del otro y la comunicación empática no son condiciones exclusivas de un sexo u otro, más bien reflejan pautas de reflexión y aprendizaje en las relaciones interpersonales.

\section{Ética del cuidado y salud sexual}

En el reporte de consulta técnica de 2002, coordinado por la Organización Mundial de la Salud, se define "salud sexual" como "un estado de bienestar físico, emocional, mental y social relacionado con la sexualidad. No es solamente la ausencia de enfermedad. La salud sexual requiere una aproximación positiva y respetuosa a la sexualidad y a las relaciones sexuales, así como la posibilidad de tener experiencias sexuales placenteras y seguras, libres de coerción, discriminación y violencia. Para que se alcance y mantenga la salud sexual, los derechos sexuales de todas las personas deben ser respetados, protegidos y cumplidos"(8).

La anterior definición se apoya en el trabajo adelantado desde la Asociación Mundial de Sexología sobre los derechos sexuales como derechos humanos, fundamentales y universales. Su declaración se produjo en el marco del Congreso Mundial de Sexología, realizado en Valencia (España) en 1997. Posteriormente, fue revisada y aprobada por la Asamblea General de la Asociación Mundial de Sexología (WAS) el 26 de agosto de 1999, durante el Congreso Mundial de Sexología en Hong Kong, República Popular China. Los derechos sexuales emanados de esta reunión son:

- Derecho a la libertad sexual. Implica la plena expresión del potencial sexual individual y excluye toda forma de coerción, explotación y abuso sexual en cualquier fase del ciclo vital.

- Derecho a la autonomía, integridad y seguridad sexuales del cuerpo. Incluye la toma de decisiones autónomas sobre la vida sexual en el contexto de la ética personal y social, así como la capacidad de control y disfrute corporal sin tortura, mutilación o cualquier otra amenaza de daño.

- Derecho a la privacidad sexual.

- Derecho a la equidad sexual. Se refiere al rechazo a cualquier forma de discriminación derivada del sexo, el género, la orientación sexual, la edad, la raza, la clase social, religión o limitación física o mental.

- Derecho al placer sexual. Alude a la obtención del placer al compartir la actividad sexual o mediante la autoestimulación.

- Derecho a la expresión sexual emocional. Toda persona tiene derecho a manifestar su sexualidad en la comunicación verbal y no verbal, el contacto, la expresión afectiva y la vinculación amorosa.

- Derecho a la libre asociación sexual. Significa la posibilidad de establecer relaciones, casarse, divorciarse y otras asociaciones sexuales.

- Derecho a la toma de decisiones reproductivas, libres y responsables. Involucra el derecho a tener hijos o no, su espaciamiento en el tiempo y la regulación de la fecundidad.

- Derecho a la información basada en el conocimiento cientifico.

- Derecho a la educación sexual integral. Comprende tanto la esfera familiar, como la escolar y la social. 
- Derecho a la atención de la salud sexual. Incluye la accesibilidad a servicios de salud idóneos para la prevención, promoción, diagnóstico, tratamiento y rehabilitación de los trastornos sexuales.

Los países de América Latina y el Caribe han presentado cifras preocupantes respecto de la prevalencia de violencia sexual, embarazos no deseados y enfermedades de transmisión sexual. Esto refleja, en último término, la necesidad generalizada de información y educación en sexualidad. También alude a la escasa importancia dada a la salud sexual como elemento constitutivo del bienestar general. La inequidad en el acceso a los servicios de salud hace que muchas niñas y mujeres no pueden obtener la atención que requieren para cuidar y recuperar su salud sexual. También, las limitaciones en actitudes y aptitudes del personal de salud hacen que no se genere una relación empática, necesaria en este proceso una vez que se han superado los problemas en el acceso. La situación de mujeres que son víctimas de violencia sexual pero que, al solicitar atención y "cuidado" en las instituciones de salud, no encuentran la respuesta adecuada hace que se reproduzca el ciclo crónico de agresión.

La ética del cuidado exige una condición básica de idoneidad y competencia técnica. Todavía se requiere que el personal de salud, en todas las áreas, asimile el cuerpo teórico y práctico que implica la promoción de la salud sexual. El desconocimiento de los derechos sexuales provoca un cumplimiento limitado. Asimismo, las comunidades que desconocen sus garantías en el ejercicio sano y placentero de la actividad sexual no se movilizan para obtener educación y otros recursos que les ayudarían a superar la brecha de inequidad en salud.

Las estructuras culturales y religiosas -rígidas e intolerantes con el placer sexual- retardan el cambio de registro respecto de la sexualidad. La salud sexual demanda la superación de mitos y creencias infundadas, tan comunes en las comunidades latinoamericanas. El problema suele tornarse más complejo cuando el mismo personal de salud se encarga de difundir y perpetuar visiones distorsionadas con relación a la experiencia sexual.

Pocos terrenos en la ciencia médica suelen tener tantas tensiones éticas como los relativos a la salud sexual y reproductiva. En los países de América Latina se encuentran, además, marcadas polarizaciones sociales respecto de temas como el aborto, la anticoncepción de emergencia (también llamada "píldora del día después"), los derechos de las personas homosexuales, el uso del condón y las estrategias para la educación sexual, por sólo mencionar algunos. ¿Qué entrenamiento ha tenido el personal de salud para resolver estas situaciones en el contexto de la atención directa? Los usuarios pueden estar convencidos de tener un derecho sexual y, al ingresar a la atención en salud, éste no es percibido como tal.

La actitud del profesional de salud es fundamental como estrategia para la difusión de los derechos sexuales y, en última instancia, para la promoción de la salud mental. Si se plantea como meta terapéutica la estructuración de la mutua confianza, como lo propone la ética del cuidado, es más probable y confiable la identificación de prácticas sexuales de riesgo y la transmisión de valores respecto del ejercicio responsable de la sexualidad.

El médico o enfermera que muestran actitud de escucha, compromiso de acción y solidaridad con el sufrimiento de sus pacientes, fomentarán la ejecución de hábitos saludables de vida. Esto debe partir no sólo desde un deber técnico relacionado con la profesión, sino desde el compromiso vital de la relación vinculante que se da entre un cuidador y su paciente. La marcada vulnerabilidad sexual de muchas adolescentes latinoamericanas puede ser aminorada si encuentran criterios claros de ayuda en las instituciones de salud. La consolidación de la cultura de la prevención en salud sexual requiere que la transmisión de los conocimientos se haga en un contexto que incluya el trato humano digno, el respeto a los valores individuales y la opción de hablar abiertamente de la sexualidad sin temor a ser sancionado o puesto en ridículo.

El interés de los usuarios por abordar sus problemas sexuales puede verse truncado si se encuentran actitudes de rechazo en el personal de salud. Estas manifestaciones recorren un espectro amplio: van desde la negación directa a cualquier mención de lo sexual hasta expresiones sutiles de sanción cuando se alega falta de tiempo. La ética del cuidado insiste en la calidad de la comunicación terapéutica: los mensajes no verbales de rechazo e, incluso, la ausencia de contacto visual también son experimentados como una sanción cuando se intenta hablar acerca de lo sexual.

Faltan estudios que analicen cómo los médicos latinoamericanos abordan las dificultades en salud sexual de 
sus pacientes a partir de su visión personal del asunto. La formación en sexología clínica implica una reflexión sobre el propio ejercicio de la sexualidad. En tal sentido, la ética del cuidado obliga a un conocimiento interior de las carencias y logros que el profesional, como ser sexual, tiene en su propia vida personal. La reflexión propuesta supera el enfoque de género: hombres y mujeres tienen por igual el compromiso de analizar cómo su propia vivencia sexual influye en la posibilidad de dar información, orientación y atención médica.

Por otro lado, los alcances técnicos en la educación sexológica suscitan la pregunta respecto de los estándares de formación en las universidades. Un profesional de la salud con una educación adecuada en lo sexual se sentirá con mayor confianza para iniciar el abordaje y remitir al especialista cuando lo considere pertinente. Desgraciadamente, el nivel de formación sexológica de los profesionales de la salud en Latinoamérica suele ser bajo.

Finalmente, conviene insistir en las múltiples opciones que puede ofrecer la reflexión desde la ética del cuidado para la promoción de la salud sexual y reproductiva. La humanización del trato a partir de la construcción de una relación empática posibilita la ejecución del arte médico como una experiencia que trasciende lo asistencial y consolida el carácter esencialmente humano de la medicina.

\section{Referencias}

1. Gracia D. Ética de los confines de la vida. Santa Fe de Bogotá: El Búho; 1998.

2. May R. Love and Hill. New York: W.W. Norton; 1969.

3. Erikson E. The Life Cycle Completed: A Review. New York: W.W. Norton; 1982.

4. Reich WT. (Ed). Encyclopedia of Bioethics. Vol. 1. New York: Macmillan; 1995.

5. Bylund CL, Makoul G. Empatic communication and gender in the physician-patient encounter. Patient Education and Counseling 2002; 48: 207-216.

6. Mathiasen H. Empathy and Simpathy: Voices from Literatura. Am J Cardiol 2006; 97: 1789-1790.

7. Gilligan C. In a Different Voice: Psychological Theory and Women's Development. Cambridge, Mass: Harvard University Press; 1982.

8. World Health Organization. Gender and reproductive rights. (Sitio en Internet) Disponible en: http://www.who.int/ reproductive-health/gender/sexual_health.html Consultada el 26 de junio de 2007.

Recibido: 18 de julio de 2007.

Aceptado: 07 de agosto de 2007. 